\title{
Implementing Mobile Virtual Exhibition to Increase Cultural Heritage Visibility
}

\author{
Cristian CIUREA ${ }^{1,2}$, Alin ZAMFIROIU ${ }^{1}$, Alin GROSU ${ }^{1}$ \\ ${ }^{1}$ The Bucharest University of Economic Studies \\ ${ }^{2}$ The Romanian Academy Library \\ cristian.ciurea@ie.ase.ro, zamfiroiu@ici.ro, alin.grosu@aptus.ro
}

The paper presents an implementation of iOS mobile application designed as a virtual exhibition, which aims to increase the accessibility and visibility of physical objects that composite cultural heritage elements. Mobile technologies have seen a huge evolution in the last years and people are very attracted by smartphones, tablets and other mobile devices. Taking into consideration the impact of mobile technologies in all the activity fields, an important research objective is to analyze the influence of mobile applications designed as virtual exhibitions on cultural heritage promotion and on people cultural needs.

Keywords: Mobile Application, Virtual Exhibition, Cultural Heritage

1 Mobile Technologies

Mobile technologies have seen a huge increase in the last period [1]. Some operating systems captured the mobile market. These are iOS, Android and Windows Phone. iOS is an operating system for mobile devices developed by Apple and distributed exclusively for Apple devices: iPhone, iPad, iPod Touch and Apple TV. Interface control elements consist of user gestures such as swipe (touch smooth), tap (simple touch), pinch (two-finger touch).

Android is an operating system for mobile devices based on the Linux kernel, developed by Google, with a direct manipulation based GUI designed primarily for mobile devices equipped with touch screen, such as smartphones and tablets. At the same time, it is used as the operating system for TVs, game consoles, navigation systems, digital cameras.

The main advantage of the Android operating system is that it is an open, free platform, due to the fact that it is based on the Java programming language, which is also open. This is the reason why the app store run by Google, called Google Play Store, comprises a total of 1.2 million applications.

Windows Phone is a mobile operating system developed by Microsoft. It is considered to be the successor to Windows Mobile, although the two versions are not compatible. Mobile applications for Windows Phone are developed using C\# programming language and development environment Visual Studio. It is used, too, C++ or HTML5 along with JavaScript.

What is new in Windows Phone is the user interface, called "Modern", in the past being known as "Metro". The first version of Windows Phone 7 was launched in 2010. Later was WP 7.5, WP 7.8, WP 8. Currently released version is WP 8.1.

Current mobile technologies presented in developing applications shows prerequisites for all existing domains.

\section{Virtual Exhibitions and Digital Exhibitions}

The term exhibition in the broad sense indicates that someone tries to expose a concept or topic explaining logical content or by reference to other concepts or topics that helps to highlight the meaning.

The exhibition term indicates an event with a specific place and time during which the public can enjoy a range of objects, paper or multimedia, books, paintings, sculptures and other objects linked together and organized according to logical criteria, thematic, space, history and made available permanently or temporarily by one or more narrative routes and having a scientific, educational and / or promotional objective [2].

The term virtual exhibition is mainly used for $3 \mathrm{D}$ reconstruction in cases, where there is a 
virtualization environment in which objects are located. Through digital exhibition is inferred that heritage items are not subject to any reconstruction process, the work of art being treated individually, possibly included in a way that treats the logical combination of materials, according to different criteria: subject, author, time, techniques.

Cultural institutions rely increasingly exhibitions leaving the traditional parameters of space and time and IT technologies provided. These can be exhibited in museums and galleries and web spaces, in which case they are called online virtual exhibitions [3]. A virtual exhibition is a collection of digital hypermedia objects that meet the following conditions:

- are linked by a common goal, an interdisciplinary topic, a concept, an idea, an anniversary, a special event or person;

- $\quad$ are presented in 2D or/and 3D;

- occasionally are stored in distributed networks;

- $\quad$ are accessible by the potential offered by modern technology, thanks to a system architecture designed to provide users new experience.

The advantages of a virtual exhibition are the followings [4], [5]:

- $\quad$ promoting cultural heritage preserved by institutions;

- serving as a learning tool that helps developing knowledge;

- $\quad$ enabling the access to a large number of documents and articles, more than any traditional exhibition could expose;

- making available to the public the most valuable artworks or documents without any risk;

- allowing users to have access to documents and papers that might not otherwise be available;

- making it possible to view certain parts and details of works that could not otherwise be seen, even by direct analysis of the original;

- remaining accessible over time, since they are not limited during the current event;
- if they are available online, they can be visited at all times by users from around the world who otherwise would not allow you to visit the exhibition;

- can be improved by users involving;

- can be arranged even with low budgets and are much cheaper than traditional exhibitions;

- can serve as an online repository for information on physical exhibition;

- can have a positive impact on the tourism industry, education, volume of sales of IT equipment and image of the country [6].

Even if sensory and emotional impact of a physical exhibition can hardly be replaced by an alternative web-based virtual experience, the added values are derived from [5]:

- opportunity to access culturally rich content directly from your personal computer or mobile phone;

- freedom to browse content whim after tracks individually determined;

- ability to save, store and reuse content for their own;

- flexibility to interconnect with other virtual exhibitions or other sites via external links [7].

Similar exhibitions can be made through mobile devices. The principle is based on the online exhibition but brings the user closer to the elements exhibit.

\section{The Development of Mobile Application}

The application architecture was designed so that the final software product to provide:

- flexibility - exhibition managers must be able to change content at any time without having to change the structure of the application; new content must be added whenever to be accessed, or to be able to modify the existing one;

- maximum accessibility - virtual exhibition must be accessed from anywhere, and web technologies provide this functionality; Internet connection is all that the user needs to enter the exhibition;

- mobility - following the current trend that we have mobile devices, more 
common among users of any age, it was concluded that a mobile application that will take the form of a virtual exhibition would be a very good solution; mobile phones provide a very high mobility, and this, together with the fact that one of the reasons why museums are visited is the lack of time, people argue the development of mobile applications.

The Figure 1 below presents the overall application architecture.

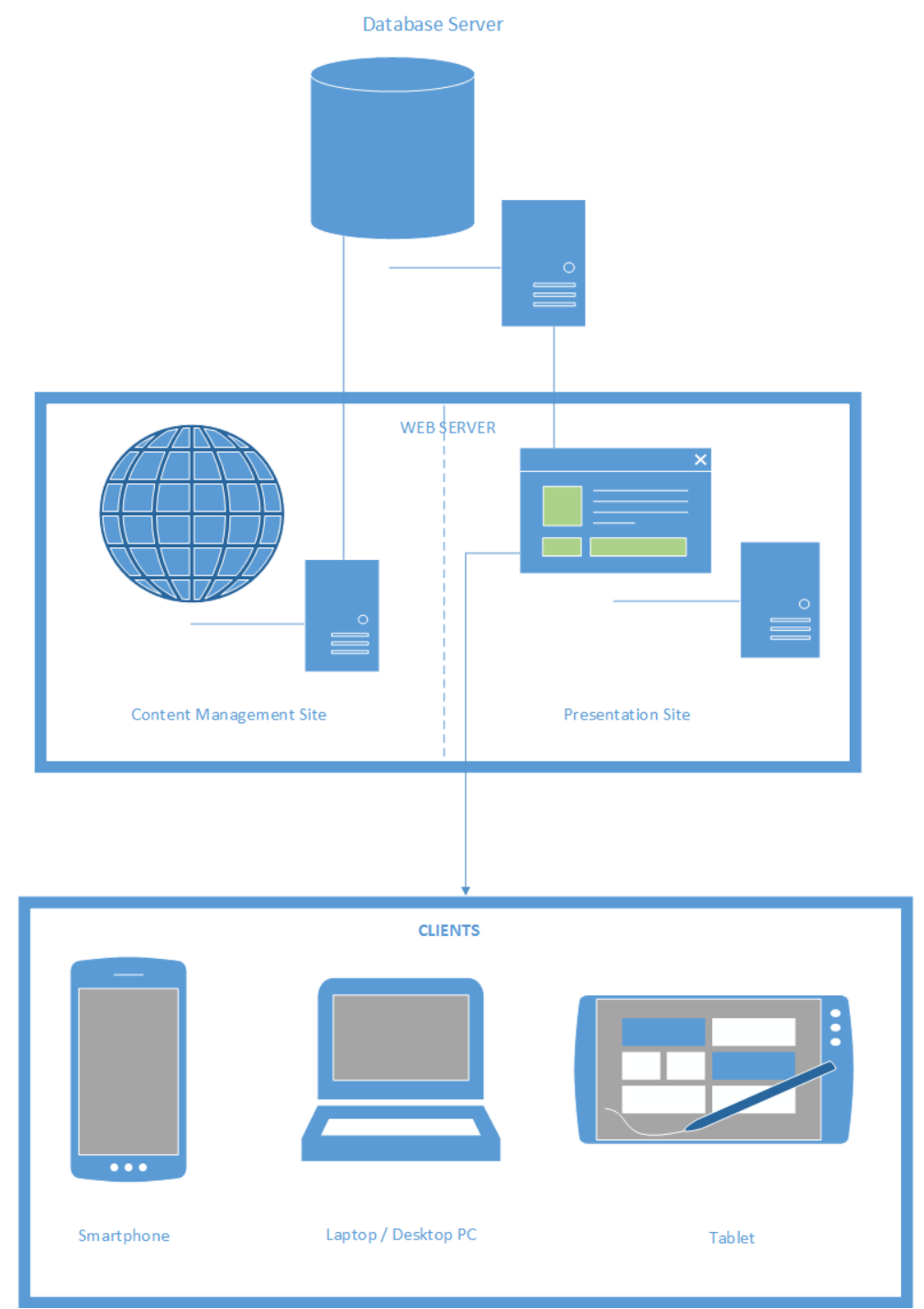

Fig. 1. Application architecture

The application architecture consists of three components:

- web content management platform;

- web platform for the presentation of the content (presentation hosting and virtual exhibition);

- mobile application for accessing the virtual exhibition.

\section{The "Smart Exhibition" Application}

In order to implement a mobile virtual exhibition to increase the national cultural heritage visibility, an iOS mobile application was developed, which contains different coins collections from the Romanian Academy Library.

The collection of coins and documents from the Romanian Academy Library is of particular interest, as they provide economic, political, social and cultural information, which can be perceived by inspecting all their constituent elements, specific to chancellery acts of the time: language, spelling, diplomatic form, autograph subscripts, seals, parchment, paper, format, size, folding, inks, script, notes on the verso, 
depending on document type or coins material [8].

The mobile application "Smart exhibition" fulfills four basic functions:

- allows viewing of coin collections by category;

- allows to save objects of interest as favorites and view them later;

- allows the use of the concept of augmented reality through Metaio SDK;

- allows recognition of QR codes (Quick Response) that may contain links to objects in the collection.

These features are implemented and structured by menu-type "side-bar". The menu opens to the left of the screen and reveals five options: home, favorites, augmented reality, $Q R$ scanner, feedback. By choosing one of the options, the user will navigate to the corresponding page. Since the mobile application was developed using the storyboard model, the navigation between pages is done using the segue links.

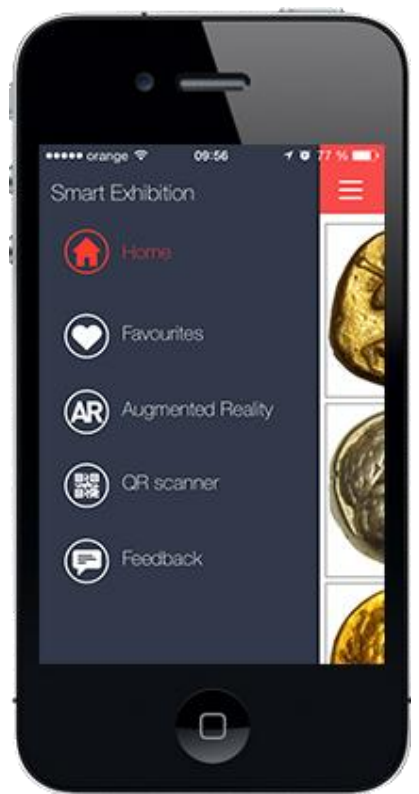

Fig. 2. Mobile application menu

The "Home" menu opens by default when launching the application. This page shows a list containing the types of coins available in gallery-items. For each category are shown: a descriptive currency type image, names and a brief description of its. This information is obtained by calling a web method that returns a JSON message. After loading the message into a data structure the category list is populated with objects derived.

Figure 2 describes the mobile application user menu.

In the Figure 3, the coins collections categories from the mobile application are presented.

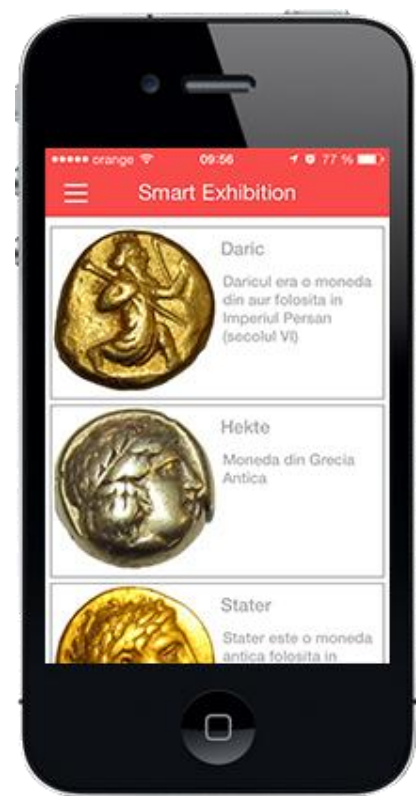

Fig. 3. Coins categories

When choosing a type of currency in the list a web method is called posing a class identifier as a parameter. The server uses LINQ to SQL language to retrieve data, based on those coins that belong to the category mentioned. The data obtained are encapsulated in a message that is sent in a JSON response. Images shown both in the category list and in the list of currencies are charged by the mobile application via a link to the resource. The images are actually stored on the web server file system in a public folder.

Figure 4 presents a list of coins in category "Stater", which are extracted from the application database.

Through some object properties, we can mention: name, description, link to the image, link to the page containing general description of the coin. By selecting a currency from the list, a browser opens an object, linked to the page containing the description of the coin. With the Angular JS web framework a slide show was created in 
this page to run automatically or manually, presenting an images gallery related to the selected currency. Also, this page shows relevant information about coin: creation date, size, description, and provider.

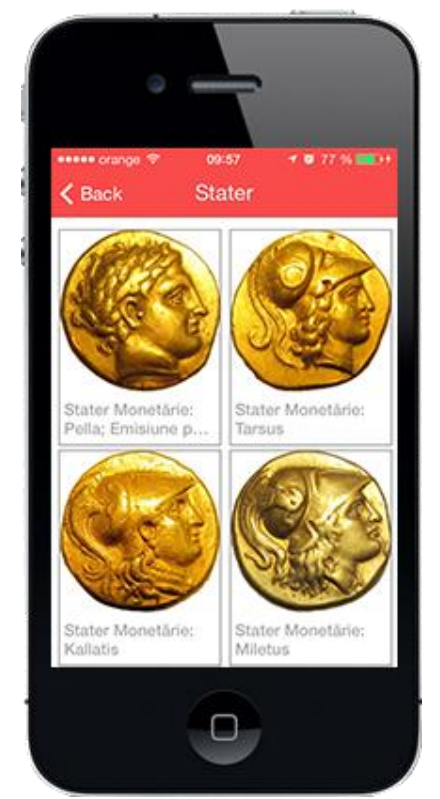

Fig. 4. List of coins in category "Stater"

Figure 5 shows the presentation view of the selected object from the list with coins in a category.

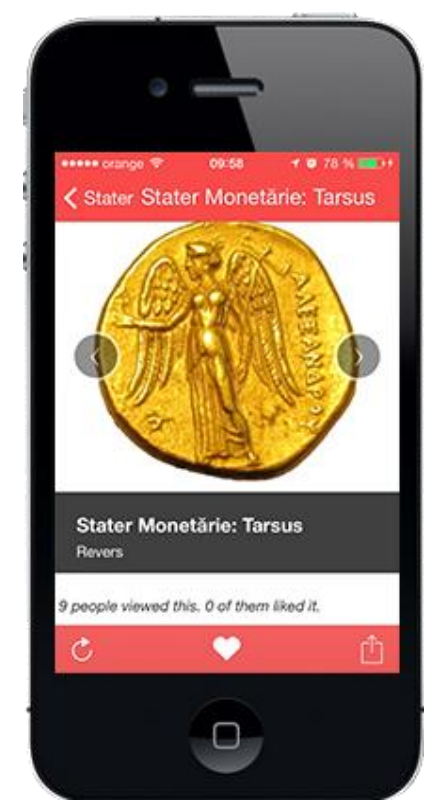

Fig. 5. Presentation of the selected object

In this page the user can save the object in a list of favorite objects, for subsequent retrieval. He also may distribute this article in social media (Facebook, Twitter), through email or via SMS. The link to the page that contains the description of the coin is distributed along with a personalized message that can be edited.

In Figure 6, the "Favorites" page is presented, which contains the coins saved by the user.

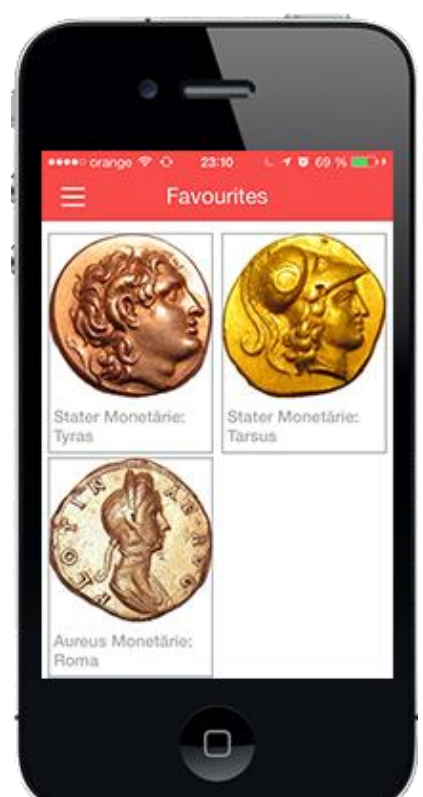

Fig. 6. The "Favorites" menu

Figure 7 shows the possibilities to distribute content from the mobile application, using social networks or personal information management facilities (email, SMS).

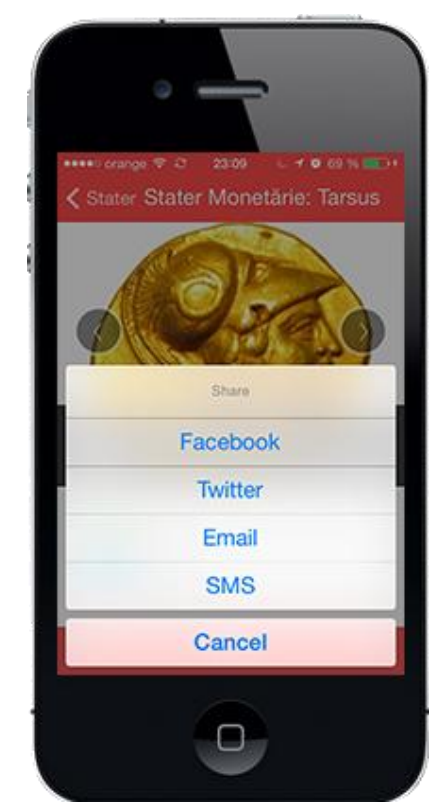

Fig. 7. Distributing content 
Later, the user can return to the "Favorites" menu, which contains the coins saved as favorite. Here, he has the opportunity to review the items or remove them from this list. To implement this functionality the device's internal memory allocated to the application was used, in which the favorites coins IDs, were saved. This ensures data persistence in the mobile application.

In the "Augmented Reality" menu, the user has the opportunity to experience this unique concept, in which, a virtual item is superimposed over the elements of reality. All you have to do is to scan the coins in collection exhibition with a mobile phone camera. At that moment, the phone will display information about the object scanned, or where applicable, its 3D projection. In terms of media content that can be displayed there are no limitations, which means that it will play video, audio, images, 3D models, 3D animations.

Figure 8 presents the augmented reality facility offered by the application.

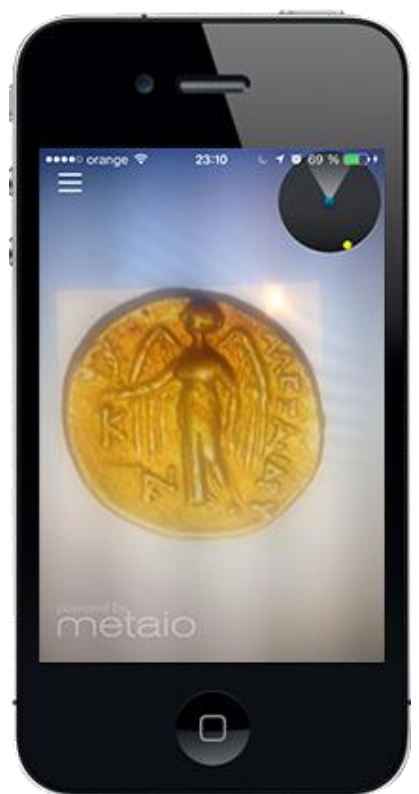

Fig. 8. Augmented reality

In Figure 9 is shown the QR codes scanning possibility offered by the mobile application, in order to easily access coins descriptions.

Through ZBAR SDK, the mobile application can read QR codes. QR codes can be generated on the basis of links to the objects in the collection, can be treated in various specialized references, documents, case studies. Through these codes descriptions of objects can be accessed easily, by simply avoiding user input errors (where the web is quite complex).

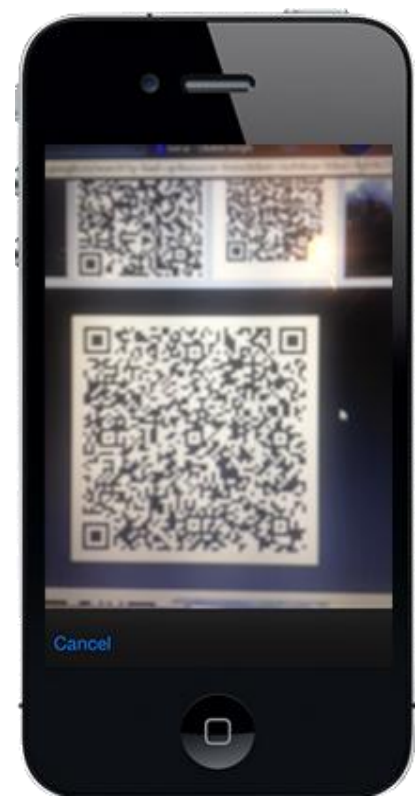

Fig. 9. Read QR codes

To keep in constant contact with application users and to receive feedback from them, it has been implemented a form through which users can make suggestions or complaints problems that they encountered in the use of application.

The feedback is encapsulated in a JSON message and sent to the server and will then be saved in the database in a dedicated table. Tracking feedback is a very important stage in the development cycle of a software application, as malfunctions can be discovered that have passed the testing stage. Also, users can provide suggestions for application development.

\section{Conclusions}

Virtual exhibitions have many advantages compared to traditional exhibitions, because it is very easy to add new components in the gallery of objects or to modify the existing ones. A particular advantage is that of having electronic duplicates of physical cultural heritage items, which in most cases are very expensive and fragile and could be damaged by handling. 
Mobile applications that take the form of virtual exhibitions and have the objective to present rare and valuable artifacts will witness a significant development in the next period, if we consider the impact they have in the educational and cultural fields. However, we believe that the market for mobile applications is increasing and the interest in such products is very high.

\section{Acknowledgement}

This paper is supported by the Sectorial Operational Programme Human Resources Development (SOP HRD), financed from the European Social Fund and by the Romanian Government under the contract number SOP HRD/159/1.5/S/136077.

\section{References}

[1] I. Ivan, D. Milodin, A. Zamfiroiu, "Studierea tipurilor de aplicatii mobile si a calitatii acestora," Revista Romana de Informatica si Automatica, vol. 21, no. 2, 2011.

[2] V. Dziekan, Virtuality and the Art of Exhibition. Curatorial Design for the Multimedia Museum, Chicago, IL: The University of Chicago Press, 2012.
[3] M. Patel, M. White, K. Walczak, P. Sayd, "Digitization to Presentation - Building Virtual Museum Exhibitions," Proceedings of International Conference on Vision, Video and Graphics, Bath, UK; Editor: Peter Hall and Phil Willis; July 2003.

[4] F. G. Filip, "Information Technologies in Cultural Institutions," Studies in Informatics and Control, vol. 6, no. 14, 1996, pp. 385-400.

[5] G. Dumitrescu, F. G. Filip, A. Ioniţă, C. Lepădatu, "Open Source Eminescu's Manuscripts: A Digitization Experiment," Studies in Informatics and Control, vol. 19, no. 1, 2010, pp. 79-84.

[6] K. Walczak, W. Cellary, "X-VRML for Advanced Virtual Reality Applications," Computer, vol. 36, no. 3, March 2003, pp. 89-92.

[7] M. T. Natale, S. Fernandez, M. Lopez (editors), Handbook on Virtual Exhibitions and Virtual Performances, version 1.0, Italy, August 2012

[8] G. Dumitrescu, C. Lepadatu, C. Ciurea, "Creating Virtual Exhibitions for Educational and Cultural Development," Informatica Economică, Vol. 18, No. 1, 2014, pp. 102-110.

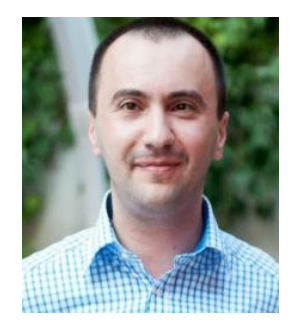

Cristian CIUREA is Assistant Professor at the Department of Economic Informatics and Cybernetics from Bucharest University of Economic Studies. He has graduated the Faculty of Economic Cybernetics, Statistics and Informatics from the Bucharest University of Economic Studies in 2007. He has a master in Informatics Project Management (2010) and a PhD in Economic Informatics (2011) from the Bucharest University of Economic Studies. Cristian has a solid background in computer science and is interested in collaborative systems related issues. Other fields of interest include software metrics, data structures, object oriented programming, windows applications programming, mobile devices programming and testing process automation for software quality assurance.

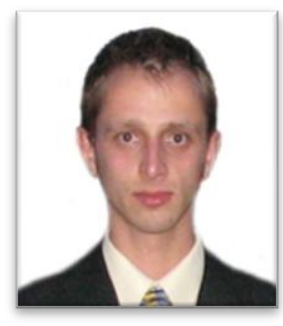

Alin ZAMFIROIU has graduated the Faculty of Cybernetics, Statistics and Economic Informatics in 2009. In 2011 he has graduated the Economic Informatics Master program organized by the Bucharest University of Economic Studies. Currently he works like a research assistant at "National Institute for Research \& Development in Informatics, Bucharest" and is $\mathrm{PhD}$ student in Economic Informatics at the Academy of Economic Studies. He has published as author and co-author of few journal articles and scientific presentations at conferences. 


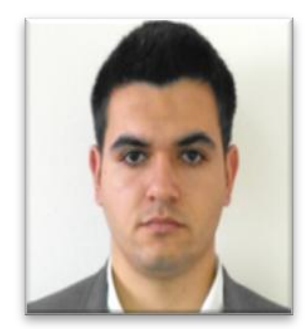

Alin GROSU has graduated the Faculty of Cybernetics, Statistics and Economic Informatics in 2012. In 2014 he has graduated the E-Business Master program organized by the Bucharest University of Economic Studies. Currently he works as Senior Software Developer in a Romanian software company, designing and developing mobile applications for iOS, Android and Windows Phone. His fields of interest include working with both Oracle and Microsoft SQL Server databases, windows applications programming and web design. 